\title{
表面改性对介孔硅酸钙镁/聚醚醚酮复合材料性能的影响
}

\author{
施张宇 ${ }^{1}$, 李 全 $^{2}$, 唐颂超 ${ }^{1}$, 钱 军 $^{1}$, 潘泳康 ${ }^{1}$, 魏 杰 $^{1}$
}

(1. 华东理工大学 教育部超细材料重点实验室, 上海 $200237 ; 2$. 第二军医大学长海医院创伤骨科, 上海 200433)

摘 要: 实验制备了介孔硅酸钙镁/聚醚醚酮复合骨修复材料, 采用砂纸打磨及喷砂对其表面进行改性。结果表明: 表面改性明显提高了复合材料表面的粗糙度和亲水性(水接触角降低), 喷砂在复合材料表面暴露出大量的介孔硅 酸钙镁, 形成了多孔结构, 粗粘度和亲水性提高最大。表面改性复合材料在模拟体液中浸泡 $7 \mathrm{~d}$ 后, 表面都形成了 大量磷灰石; 表面改性促进了 MC3T3-E1 细胞在复合材料表面粘附、增殖和分化。喷砂比砂纸打磨更明显地提高 了复合材料的生物学性能。

关 键 词: 介孔硅酸钻镁; 聚醚醚酮; 复合材料; 表面改性; 表面性能 中图分类号: TB383 文献标识码: A

\section{Surface Modification on Property of Mesoporous Calcium Magnesium Silicate/Polyetheretherketone Composites}

\author{
SHI Zhang-Yu ${ }^{1}$, LI Quan ${ }^{2}$, TANG Song-Chao ${ }^{1}$, QIAN Jun ${ }^{1}$, PAN Yong-Kang ${ }^{1}$, WEI Jie ${ }^{1}$
}

(1. Key Laboratory for Ultrafine Materials of Ministry of Education, East China University of Science and Technology, Shanghai 200237, China; 2. Department of Orthopaedics Trauma, Changhai Hospital, Second Military Medical University, Shanghai 200433, China)

\begin{abstract}
Mesoporous calcium magnesium silicate (m-MCS)/polyetheretherketone (PK) bioactive composites for bone repair were prepared, and the surfaces of composites were modified by sanding and sandblasting treatment. The surface roughness of the composites modified by sandblasting was $R_{\mathrm{a}}=4.552$ while the surface roughness of the composites modified by sanding were 1.727 and 2.103 , which were higher than that of the untreated composites (1.359). In addition, the water contact angle of the composites treated by sandblasting was $54.1^{\circ}$ while the water contact angles of the composites modified by sanding were $76.3^{\circ}$ and $71.6^{\circ}$, which were higher than that of the untreated composites $\left(81.2^{\circ}\right)$. The results indicated that surface roughness and hydrophilicity of composites were significantly enhanced by sandblasting treatment. Porous surface structure and a large number of mesoporous calcium magnesium silicates were exposed on the composites surfaces after sandblasting treatment, resulted in the maximum surface roughness and hydrophilicity. After immersed in simulated body fluid (SBF) solution for $7 \mathrm{~d}$, massive apatites were formed on the composites surfaces, showing a good bioactivity. The in vitro cell experiments indicated that the surface modified composites could significantly promote adhesion, proliferation and differentiation of MC3T3-E1 cells on the surfaces. Therefore, sandblasting treatment could significantly improve the biological performances of the composites as compared with sanding treatment.
\end{abstract}

Key words: mesoporous calcium magnesium silicate; polyetheretherketone; composite; surface modification; surface properties

收稿日期: 2017-03-15; 收到修改稿日期：2017-05-05

基金项目: 国家重点研发计划(2016YFC1102100)

National Key Research and Development Program (2016YFC1102100)

作者简介: 施张宇(1991-), 男, 硕士研究生. E-mail: 489365696@qq.com

通讯作者: 魏 杰, 研究员. E-mail: jiewei7860@sina.com; 潘泳康, 副教授. E-mail: ykpan@ecust.edu.cn 
聚醚醚酮 $(\mathrm{PK})$ 具有优良的生物相容性和力学性 能, 且弹性模量与人体骨相近, 作为骨修复材料, 已在临床广泛应用 ${ }^{[1]}$ 。但 PK 是生物惰性材料, 植入 体内, 易被纤维组织包裹, 不能与骨形成牢固的骨 性结合, 导致植入体松动, 甚至脱落 ${ }^{[2]}$ 。生物活性材 料如生物玻璃、羟基磷灰石等, 植入体内, 可与骨形 成骨性结合 ${ }^{[3-4]}$, 因此很多研究将生物活性材料添 加到聚合物基体中, 制成生物活性无机/有机复合骨 修复材料 ${ }^{[5-6]}$ 。介孔硅酸钙镁 (m-MCS) 是一种新型生 物活性材料, 具有较大的比表面积和孔容, 在体外 模拟体液 $(\mathrm{SBF})$ 中能够快速地形成磷灰石; m-MCS 可以刺激成骨细胞增殖与分化, 并能促进新骨再 生 ${ }^{[7-8]}$ 。因此, 本研究将 $\mathrm{m}-\mathrm{MCS}$ 添加到 $\mathrm{PK}$ 基体中, 制备 MPC 骨修复材料。另外, 植入材料的表面性能 对细胞行为有很重要的影响, 如亲水性、粗糙度、 多孔性 ${ }^{[9-10]}$ 等。因此, 本研究采用砂纸打磨及喷砂对 MPC 材料表面进行改性, 进一步提高其表面生物学 性能。

\section{1 实验方法}

\section{1 介孔硅酸钙镁的合成}

将 $6.6 \mathrm{~g}$ 十六烷基三甲基溴化铵 $(\mathrm{CTAB}$ ，上海凌 峰化学试剂有限公司)溶解在 $500 \mathrm{~mL}$ 去离子水中, 搅拌至澄清, 加入 $12 \mathrm{~mL}$ 氨水 $\left(\mathrm{NH}_{3} \cdot \mathrm{H}_{2} \mathrm{O}\right.$, 国药集团 化学试剂有限公司)使溶液呈碱性, 逐滴添加 $27.9 \mathrm{~g}$ 正硅酸乙酯(TEOS, 国药集团化学试剂有限公司), 待均匀分散后, 将 $15.8 \mathrm{~g}$ 四水硝酸钲 $\left(\mathrm{Ca}\left(\mathrm{NO}_{3}\right)_{2} \cdot 4 \mathrm{H}_{2} \mathrm{O}\right.$, 上海凌峰化学试剂有限公司)和 $17.2 \mathrm{~g}$ 六水硝酸美 $\left(\mathrm{Mg}\left(\mathrm{NO}_{3}\right)_{2} \cdot 6 \mathrm{H}_{2} \mathrm{O}\right.$, 上海凌峰化学试剂有限公司)加 入到溶液中, 在 $50^{\circ} \mathrm{C}$ 下, 摚拌反应 $4 \mathrm{~h}$, 静置 $16 \mathrm{~h}$, 分别用去离子水和无水乙醇清洗三次, 再将烘干后 的样品置于马弗炉中高温陆烧 $\left(600^{\circ} \mathrm{C}, 360 \mathrm{~min}\right.$, 升 温速率为 $1^{\circ} \mathrm{C} / \mathrm{min}$ ), 得到 $\mathrm{m}-\mathrm{MCS}$ 。

将 m-MCS 研磨成粉末, 在无水乙醇中超声分 散 $15 \mathrm{~min}$, 用吸管取一滴溶液滴在铜网上, 干燥后 用透射电子显微镜(TEM, JEM1400F 型，日本 JEOL 公司)观察 m-MCS 的微观结构和形貌。采用能谱仪 (EDS, QUANTAX, 布鲁克 AXS 有限公司)分析 mMCS 的元素组成和含量。

\section{2 复合材料的制备及表面改性}

将 m-MCS 粉末和 PK(国药集团化学试剂有限 公司)粉末按一定质量比(m-MCS : $\mathrm{PK}=2: 3$ )放入高 速球磨机中混合 $1 \mathrm{~h}$, 随后在 $150^{\circ} \mathrm{C}$ 烘干。将混合物 放入模具中, 用压片机压制成 $\phi 12 \mathrm{~mm} \times 2 \mathrm{~mm}$ 圆片。
再将原片样品放入马弗炉中烧结 $\left(350^{\circ} \mathrm{C}\right)$, 烧结时间 为 $120 \mathrm{~min}$ 。分别采用 $800 \#$ (MPC8)和 400\#(MPC4) 砂纸打磨复合材料表面，采用喷砂处理复合材料表 面(MPCsb) (喷砂颗粒为氧化铝，砂粒的粒径 $180 \mu \mathrm{m}$, 压力为 $0.5 \mathrm{MPa}$, 时间 $60 \mathrm{~s})$, 未表面处理的复合材 料为对照样品(MPC)。

\section{3 表面改性复合材料的表征}

将 MPC、MPC8、MPC4 和 MPCsb 四组样品超 声清洗后干燥, 在扫描电子显微镜(SEM, S-3400 型, 日本 JEOL 公司)下观察材料的表面形貌; 采用 $X$ 射 线衍射仪(D/max $2550 \mathrm{VB} / \mathrm{PC}$ 型，日本 Rigaku 公司) 对样品进行分析; 采用激光共聚焦 $3 \mathrm{D}$ 显微镜 (LEXT OLS4000, 上海舟超光电科技有限公司)观 察样品表面形貌, 并采集 2D 与 3D 图像, 同时获得 材料表面的粗粘度 ( $R_{\mathrm{a}}$ 值); 采用水接触角测量仪 (JCY 型，上海方瑞仪器有限公司)测试样品的亲水性。

\section{4 表面改性复合材料的体外生物活性}

将表面改性复合材料浸泡在模拟体液 (SBF, $\mathrm{pH}=7.4)$ 中，材料与液体的质量比为 $1 / 20(\mathrm{~g} / \mathrm{mL})$, 分 别在不同时间点取 $1 \mathrm{~mL}$ 浸泡液，用等离子体发射 光谱仪(ICPE-9000)测试溶液中 $\mathrm{Ca} 、 \mathrm{P}$ 和 $\mathrm{Si}$ 离子浓 度, 浸泡 $7 \mathrm{~d}$ 后, 将样品取出, 用 SEM 观察其表面 微观形貌, 用 EDS 分析样品表面沉积物的元素组成 和含量。

\section{5 细胞实验}

将四组样品高温灭菌后, 置于 24 孔培养板中, 在 样品表面接种 MC3T3-E1 细胞(密度为 $2.5 \times 10^{4} \mathrm{~mL}^{-1}$, 中国科学院细胞库), 细胞培养环境为 $37.5^{\circ} \mathrm{C} 、 95 \%$ 相对湿度和 $5 \% \mathrm{CO}_{2}$ 气氛。在不同时间点，用磷酸盐 缓冲液(PBS)清洗样品表面, 再用 $2.5 \%$ 的戊二醛溶 液固定细胞, 分别用鬼笔环肽(FITC, GIBCO 公司) 和 DAPI (Songon 公司)对细胞的细胞质和细胞核染 色，通过激光共聚焦显微镜(CLSM，Nikon AIR 型, 日本 Nikon 公司)观察细胞形貌。

按照上述方法将 MC3T3-E1 细胞接种于样品表 面, 培养 $1 、 3$ 和 $7 \mathrm{~d}$ 后, 将样品转入新的培养板中, 加入 $500 \mu \mathrm{L} \mathrm{CCK}-8$ 试剂(GIBCO 公司), 继续培养 $4 \mathrm{~h}$ 后, 吸取 $10 \mu \mathrm{L}$ 培养液, 用酶标仪在 $\lambda=490 \mathrm{~nm}$ 处测 试样品表面的光密度值(OD), 研究细胞在材料表面 的增殖情况。

细胞培养方法同上, 分别在 7、10 和 $14 \mathrm{~d}$, 用 PBS 缓冲液清洗样品, 加入 $500 \mu \mathrm{L}$ 浓度为 $1 \%$ 的乙 基苯基聚乙二醇溶液(Nonidet P-40, GIBCO 公司), 获得细胞裂解液。随后每孔加入 $50 \mu \mathrm{L}$ 的 $\mathrm{p}$-硝基苯 磷酸盐溶液(pNPP, 浓度为 $1 \mathrm{mg} / \mathrm{mL}$, GIBCO 公司), 
室温下放置 $15 \mathrm{~min}$ 。最后加入 $100 \mu \mathrm{L} 0.1 \mathrm{~mol} / \mathrm{L}$ 的 $\mathrm{NaOH}$ 溶液终止反应。用酶标仪在 $\lambda=405 \mathrm{~nm}$ 处测试 OD 值, 根据 OD 值计算细胞的 ALP 活性, 研究细胞 在材料表面的成骨分化情况。

\section{6 统计方法}

所有数据均通过 Origin Pro 8 (Origin Lab Corporation, USA)软件处理, 并以(平均值土标准差)的 形式表示。 $P>0.05$ 表示实验所获得的数据无显著性 差异, 不具有统计学意义; $P<0.05$ 表示实验所获得 的数据具有显著性差异。

\section{2 结果与讨论}

\section{$2.1 \mathrm{~m}-M C S$ 的 TEM 和 EDS 分析}

图 1(a)是 m-MCS 的 TEM 照片, 从图中可以看 出, m-MCS 为球形颗粒, 粒径在 $200 \mathrm{~nm}$ 左右; $\mathrm{m}$ MCS 内部有介孔结构。m-MCS 的 EDS 图谱见图 1(b), 从图中可以看出, $\mathrm{m}-\mathrm{MCS}$ 含有 $\mathrm{Ca}, \mathrm{Mg}$ 和 $\mathrm{Si}$ 三种元素, 且元素的原子含量比近似为 $\mathrm{Mg}: \mathrm{Ca}: \mathrm{Si} \approx 1: 1: 2$ 。

\section{2 表面改性对复合材料表面形貌和组成的 影响}

图 2 为表面改性复合材料的 SEM 照片和 XRD 图谱。由图 2(a)可知, MPC 的表面比较平整, 表面 m-MCS 颗粒少, 可能被 PK 层包裹其中。图 2(b) 和 2(c)是 MPC8 和 MPC4 的表面形貌, 由图可见, 经砂 纸打磨后, MPC8 和 MPC4 表面形成了不同程度的


图 1 m-MCS 的 TEM 照片(a)和 EDS 图谱(b)

Fig. 1 TEM image (a) and EDS spectrum (b) of m-MCS
沟槽, 表面粗糙度增加(相比 MPC), 并且经砂纸打 磨后, 复合材料表面暴露出大量的 m-MCS 颗粒。图 2(d) 是喷砂处理的复合材料表面, 由图可见, 复合 材料表面有很多凹坑，形成了多孔粗糙表面。喷砂 与砂纸打磨后, 复合材料的表面形貌明显不同, 这 是因为砂纸打磨掉了复合材料表面存在的 PK 层, 暴露出大量的 m-MCS 颗粒; 而喷砂是用陶瓷颗粒 撞击复合材料表面, 在其表面形成了多孔结构, 并 暴露出更多 m-MCS 颗粒。

图 2(e) 是 MPC, MPC $8, M P C 4$ 和 MPCsb 的 XRD 图谱, 可以看出, 复合材料有 4 个特征峰, 分别在 $2 \theta=18.9^{\circ} 、 22.9^{\circ} 、 23.1^{\circ}$ 和 $29.1^{\circ}$, 这是 PK 的特征峰 ${ }^{[11]}$, 而 m-MCS 呈无定形态, 无明显衍射峰 ${ }^{[12]}$ 。复合材 料表面经砂纸打磨和喷砂后，尽管有大量的 $\mathrm{m}-\mathrm{MCS}$ 暴露在表面, 但复合材料的组成没有发生 变化，因而 $\mathrm{m}-\mathrm{MCS}$ 和 $\mathrm{PK}$ 的特征峰并没有发生变化， 表面处理不改变复合材料基体的组成。

\section{3 表面改性对复合材料表面粗楉度和亲水 性的影响}

图 3 为表面改性复合材料的激光显微镜 $2 \mathrm{D}$ 照 片。MPC 的表面包裹了一层 PK, 表面 m-MCS 颗粒 少(图 3(a))。MPC8 和 MPC4 表面覆盖的 PK 层被磨 除，原本平整的表面变得粗糙，暴露出 m-MCS 颗粒 (图 3(b)和 3(c)); MPC4 表面明显比 MPC8 粗糙, 说 明打磨砂纸的粒度粗细影响材料表面粗鋉度。 MPCsb 的表面形成了很多凹坑和沟壑，表面非常粗 粮，暴露出大量 m-MCS 颗粒(图 3(d))。

图 4 是表面改性复合材料的激光显微镜 3D 照 片。MPC 的表面比较平整, 但有一定程度的起伏(图 4(a))。MPC8 和 MPC4 表面出现了一些小的沟壑, MPC4 表面的沟壑比 MPC8 明显, 说明打磨砂纸的 粒度粗细影响复合材料表面沟壑的形貌(图 4(b)和 $4(\mathrm{c}))$ 。 MPCsb 的表面变得更加粗糙, 沟壑深而多, 形状不一(图 4(d))。

表 1 是表面改性复合材料的粗糙度 $R_{\mathrm{a}}$ 值, 由表 1 可知, MPC 的粗粘度最低(1.359), 而 MPC8 和 MPC4 表面粗粘度分别是 1.727 和 2.103, 说明砂纸 打磨后，复合材料表面的粗粘度变大，且随砂纸粒 度增大而变大，而喷砂处理的复合材料表面的粗糙 度为 4.552 , 明显提高。结果表明: 复合材料表面经 改性后，粗粘度都明显提高，其中喷砂处理的材料 表面粗粘度提高最大。另外, 复合材料经表面改性 后，其表面亲水性也发生了明显改变, MPC、MPC8、 MPC4 和 MPCsb 的水接触角分别为 $81.2^{\circ} 、 76.3^{\circ}$ 、 $71.6^{\circ}$ 和 $54.1^{\circ}$, 这是因为表面改性使复合材料表面 

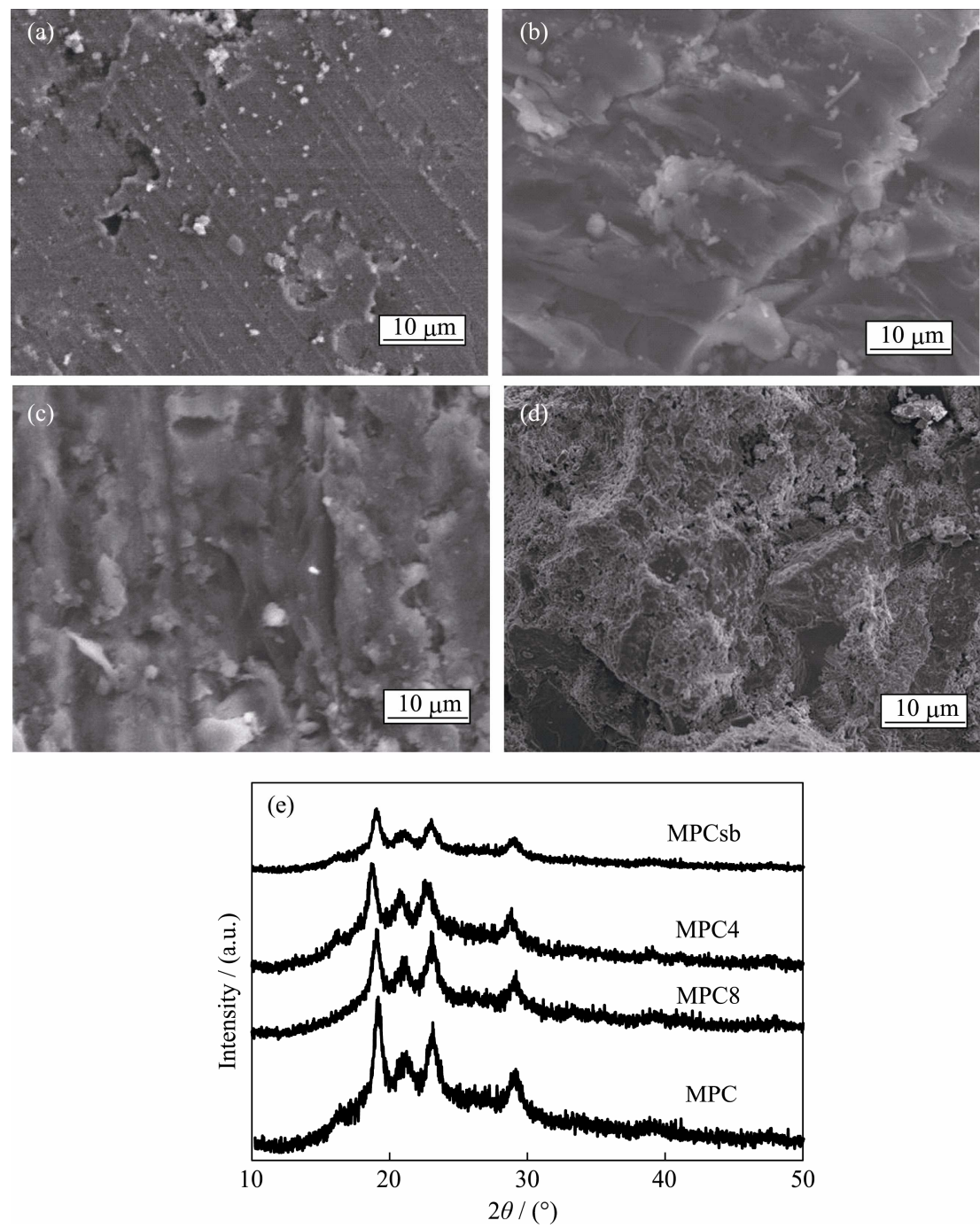

图 2 表面改性复合材料的 SEM 照片((a) MPC; (b) MPC8, (c) MPC4; (d) MPCsb)和 XRD 图谱(e)

Fig. 2 SEM images ((a) MPC; (b) MPC8; (c) MPC4; (d) MPCsb) and XRD patterns (e) of the composites after surface modification
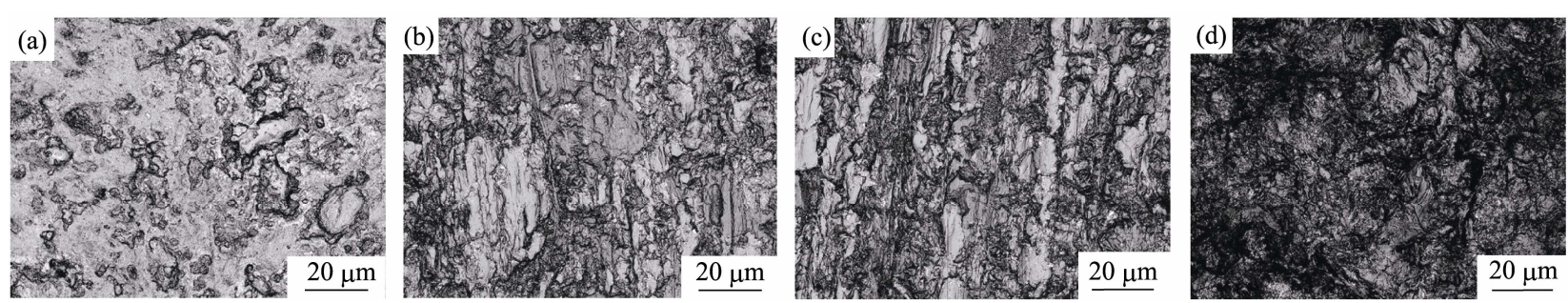

图 3 表面改性复合材料的激光显微镜 $2 \mathrm{D}$ 照片

Fig. 3 Laser microscope 2D images of the surface modified composites (a) MPC; (b) MPC8; (c) MPC4; (d) MPCsb

不同程度地暴露出大量的 m-MCS 颗粒, 材料表面 暴露的 m-MCS 颗粒越多，其表面亲水性越好 ${ }^{[13]}$ 。

\section{4 表面改性对复合材料体外生物活性的影响}

图 5 为表面改性复合材料在 SBF 中浸泡 $7 \mathrm{~d}$ 的 SEM 照片和喷砂表面的 EDS 图谱。在 SBF 中矿化 $7 \mathrm{~d}$, 复合材料表面均形成了磷灰石。MPC 表面的磷 灰石最少(图 5(a)), 这是由于 MPC 表面只有少量 m-MCS, 不利于其在 SBF 中形成磷灰石, 说明未改
性复合材料表面的生物活性最差。经砂纸打磨后, 复合材料表面在 SBF 中生成了较多的磷灰石(图 5(b), (c)), MPC4 表面磷灰石比 MPC8 多, 说明用粒 度大的砂纸打磨后，复合材料表面暴露的 m-MCS 多, 并且其表面粗糙度也越大, 有利于磷灰石沉积。 而经喷砂处理的复合材料表面被更多的磷灰石覆盖 (图 5(d)), 由于经喷砂处理后, 复合材料表面的粗糙 度最大, 其表面暴露出大量的 m-MCS, 有利于形成 

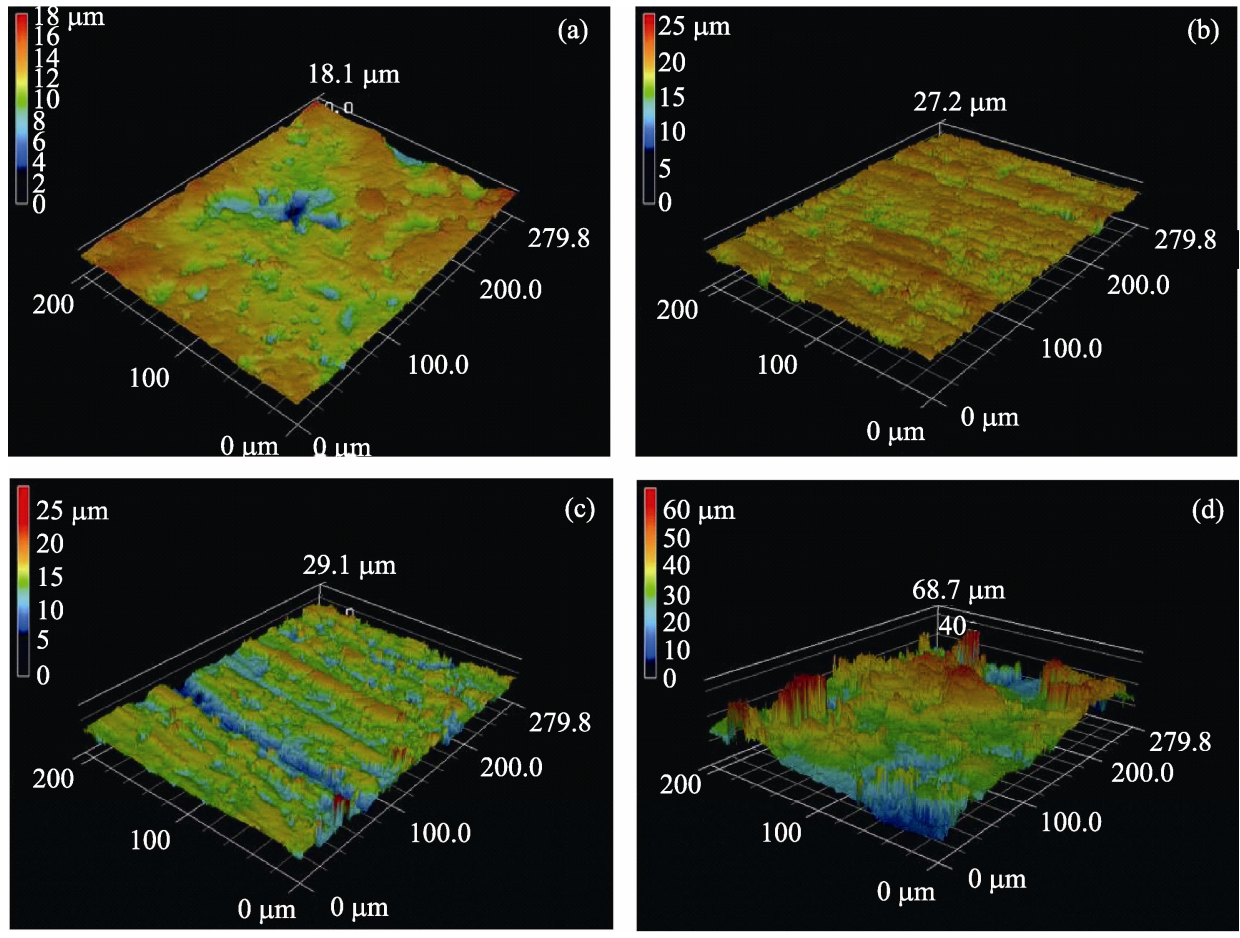

图 4 表面改性复合材料的激光显微镜 3D 照片

Fig. 4 Laser microscope 3D images of the surface modified composites (a) MPC; (b) MPC 8; (c) MPC4; (d) MPCsb

表 1 表面改性复合材料的表面粗粮度和水接触角

Table 1 Surface roughness and water contact angle of the surface modified composites

\begin{tabular}{ccc}
\hline Samples & $R_{\mathrm{a}} / \mu \mathrm{m}$ & Water contact angle $/\left(^{\circ}\right)$ \\
\hline MPC & 1.359 & 81.2 \\
MPC8 & 1.727 & 76.3 \\
MPC4 & 2.103 & 71.6 \\
MPCsb & 4.552 & 54.1 \\
\hline
\end{tabular}

大量磷灰石 ${ }^{[14-15]}$ 。图 5(e) 是 MPCsb 在 SBF 浸泡 $7 \mathrm{~d}$ 的 EDS 图谱，出现了 $\mathrm{Ca} 、 \mathrm{P}$ 特征峰，表明复合材料 表面的沉积物是磷灰石。m-MCS 具有较大的比表面 积和孔容, 其在 SBF 中能快速形成磷灰石, 具有很 好的生物活性 ${ }^{[16]}$ 。

图 6 为表面改性复合材料在 SBF 中浸泡不同时 间后，溶液中 $\mathrm{Ca} 、 \mathrm{P} 、 \mathrm{Si}$ 离子浓度变化。从图 6 可 知, $\mathrm{Ca}$ 离子先升后降, $\mathrm{P}$ 离子随时间逐渐降低, $\mathrm{Si}$ 离 子逐渐上升。这是因为复合材料经表面处理后，暴 露出的 m-MCS 在 SBF 中溶解, 释放少量的 $\mathrm{Ca}$ 和 $\mathrm{Si}$ 离子。同时, 复合材料表面形成磷灰石需要消耗 一些 $\mathrm{Ca}$ 和 $\mathrm{P}$ 离子, 因此, $\mathrm{Ca}$ 与 $\mathrm{P}$ 离子浓度随浸泡 时间而降低。在矿化前期, 形成磷灰石消耗的 $\mathrm{Ca}$ 离子要少于材料表面释放的 $\mathrm{Ca}$ 离子, 溶液中的 $\mathrm{Ca}$ 离子浓度先增后降。表面未处理的复合材料生 成少量磷灰石, 因而 $\mathrm{Ca} 、 \mathrm{P} 、 \mathrm{Si}$ 离子浓度变化较 小。在 SBF 中, 通过离子溶解一沉积过程, 复合材
料表面形成磷灰石 ${ }^{[15]}$ 。

\section{5 复合材料表面改性对成骨细胞行为的影响}

图 7 为 MC3T3-E1 细胞在表面改性复合材料表 面培养 $72 \mathrm{~h}$ 后的 CLSM 图片, 由图可见, 细胞在几 种材料表面铺展得良好, 且细胞骨架轮廓清晰, 说 明几种复合材料都具有优良的生物相容性。与 MPC 相比, MPC8 和 MPC4 表面的细胞数量明显增多(图 7(a),(b),(c))，这说明复合材料经砂纸打磨后，其表 面粗糙度和亲水性增加, 这些表面特征有利于细胞 在材料表面的粘附和生长 ${ }^{[17]}$ 。图 7(d)显示, 细胞在 MPCsb 表面最多, 说明经喷砂处理的复合材料表面 具有多孔结构, 以及粗粮度和亲水性最大的表面特 征有利于细胞在材料表面的粘附和生长 ${ }^{[17]}$ 。

图 8(A)为 MC3T3-E1 细胞在材料表面培养不同 时间的细胞增殖情况，由图可见，细胞在 MPC 上的 增殖率最低 $(1 、 3 、 7 \mathrm{~d})$; 培养的第 3 和 7 天, 细胞在 MPC8 和 MPC4 上的增殖率明显高于 MPC, 说明复 合材料经砂纸打磨后, 其表面粗䊁度和亲水性增加, 不仅有利于细胞的粘附, 而且有利于细胞增殖 ${ }^{[17]}$ 。 培养的第 3 和 7 天, 细胞在 MPCsb 上的增殖率明显 高于 MPC8 和 MPC4, 这是由于喷砂处理后的复合 材料表面的粗䊁度和亲水性更大，而且形成了多孔 结构, 这些表面特征既有利于细胞的粘附, 又有利 于细胞增殖 ${ }^{[18]}$ 。研究表明: 生物玻璃或硅酸钙在溶 液中释放出适当浓度的钙、硅离子可以促进细胞增 

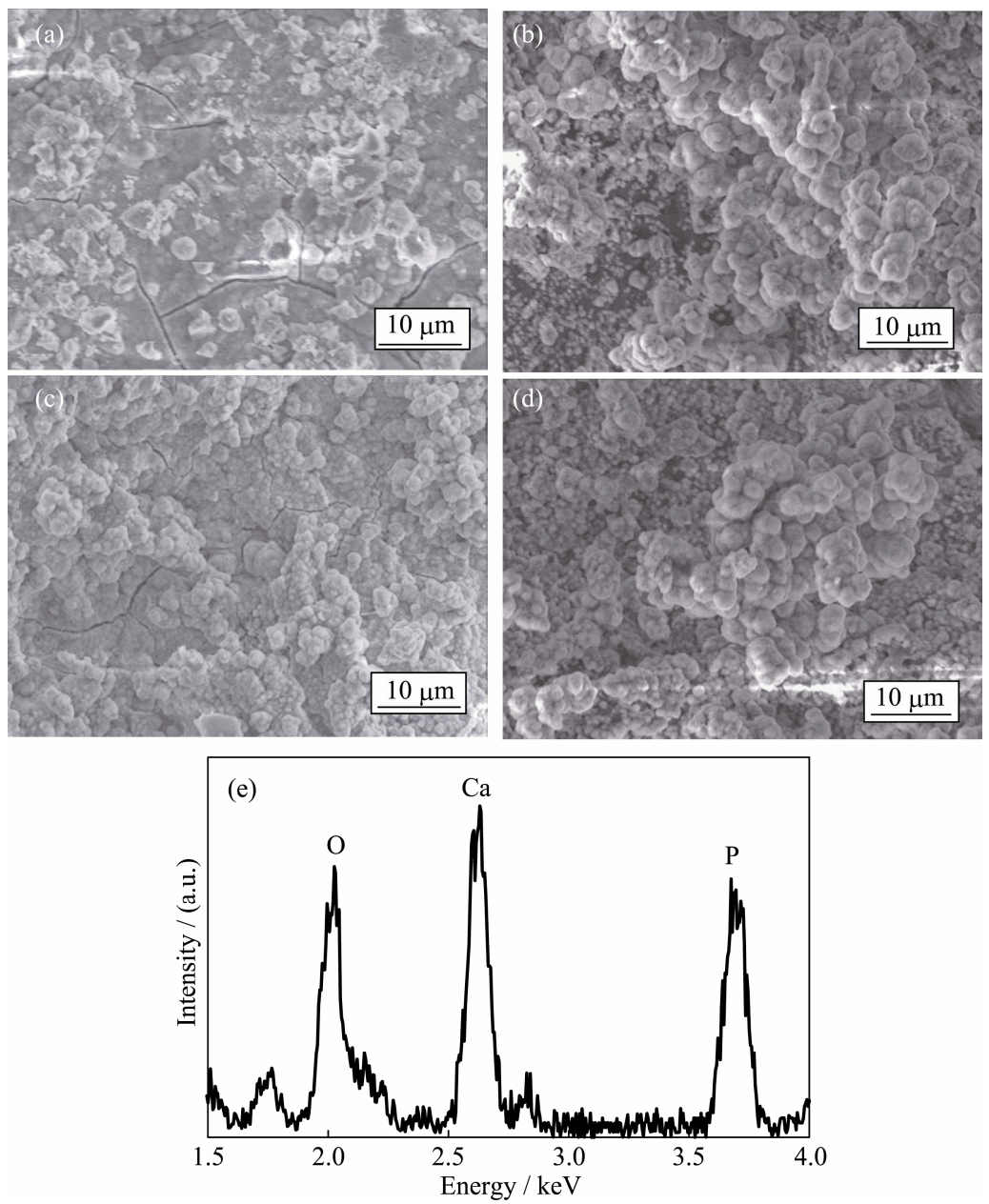

图 5 表面改性复合材料在 SBF 中浸泡 7d 的 SEM 照片

(a) MPC; (b) MPC8; (c) MPC4; (d) MPCsb 和 MPCsb 表面矿化的 EDS 图谱(e)

Fig. 5 SEM images of the surface modified composites soaked in SBF for $7 \mathrm{~d}$

(a) MPC; (b) MPC8; (c) MPC4; (d) MPCsb and (e) EDS spectrum of MPCsb
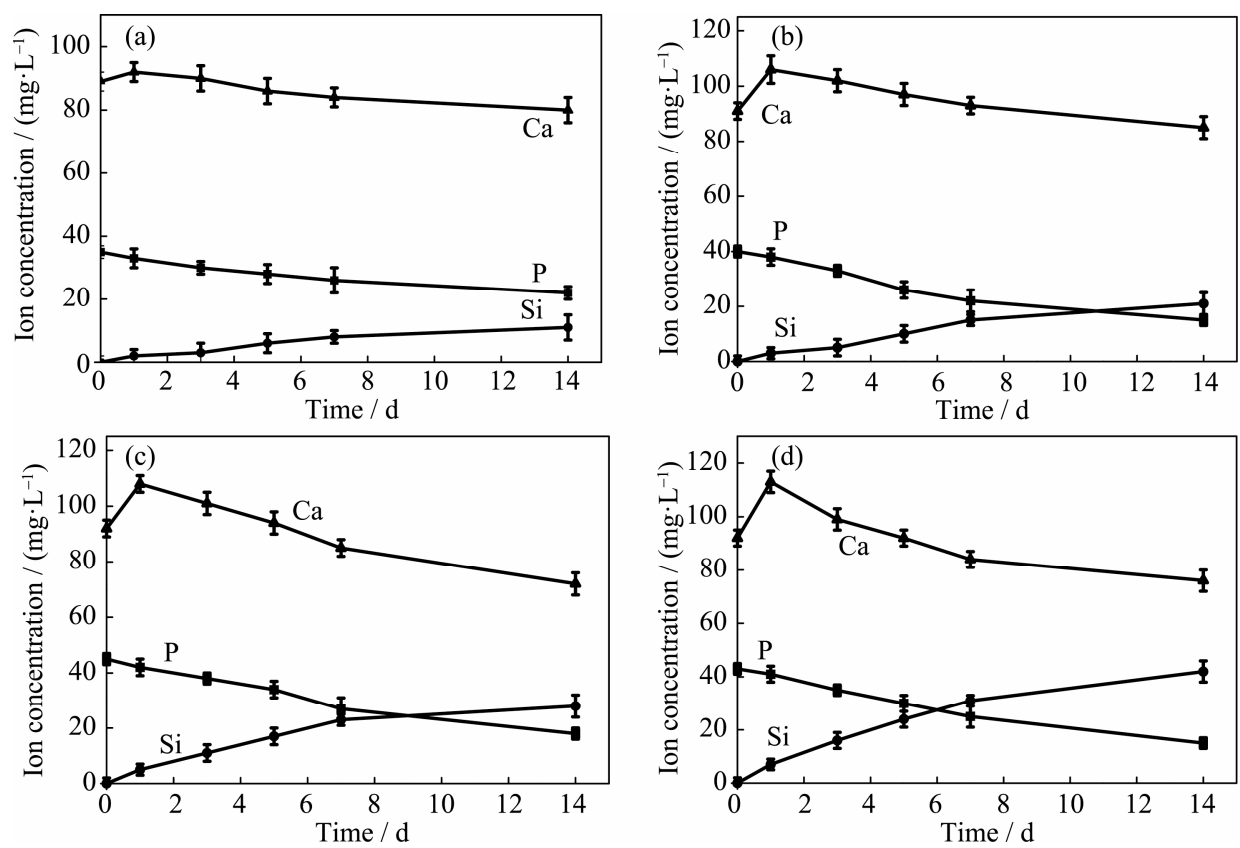

图 6 表面改性复合材料在 $\mathrm{SBF}$ 中浸泡不同时间的离子 $(\mathrm{Ca}, \mathrm{P}$ 和 $\mathrm{Si})$ 浓度变化

Fig. 6 Changes of ion (Ca,P and Si) concentrations for the surface modified composites soaked in SBF solution for different periods (a) MPC; (b) MPC8; (c) MPC4; (d) MPCsb 



图 7 MC3T3-E1 细胞在表面改性复合材料培养 $72 \mathrm{~h}$ 的激光共聚焦照片

Fig. 7 CLSM photos of MC3T3-E1 cells cultivated on the surface modified composites for $72 \mathrm{~h}$ (a) MPC; (b) MPC8; (c) MPC4; (d) MPCsb

殖 ${ }^{[19]}$ 。通过表面改性，更多的 m-MCS 暴露在复合 材料表面, m-MCS 在细胞培养液溶解, 并释放出 钻、硅离子，这些离子促进了细胞增殖。

ALP 是成骨细胞分化的标志酶, ALP 活性的高 低代表了成骨细胞分化程度 ${ }^{[20]}$ 。从图 8(B) 可以看出, 培养的第 $7 \mathrm{~d}, 4$ 组复合材料的细胞 ALP 活性无明显 区别。随着时间的延长, ALP 活性逐步升高, 表明成 骨细胞分化, 培养的第 $10 \mathrm{~d}, \mathrm{MPCsb}$ 表面的细胞 ALP 活性高于其它 3 组，表明 MPCsb 促进了成骨细 胞分化。培养的第 $14 \mathrm{~d}$, 表面改性复合材料上的细 胞 ALP 活性明显高于 MPC, 说明表面改性复合材 料促进了成骨细胞分化。研究表明: 生物活性材料 表面形成磷灰石有利于成骨细胞分化。在本研究中,
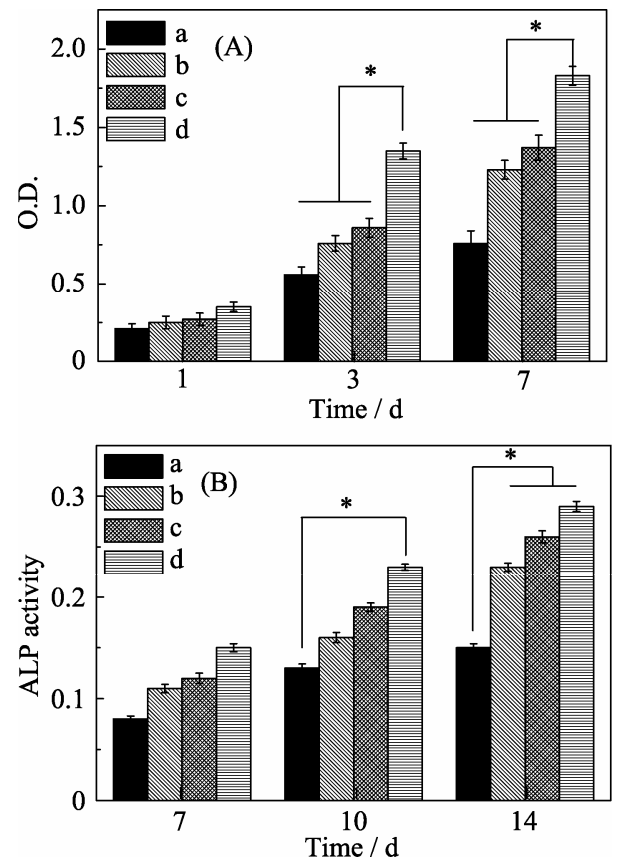

图 8 MC3T3-E1 细胞在表面改性复合材料((a) MPC; (b) MPC8; (c) MPC4; (d) MPCsb)培养不同时间的细胞增殖(A)和 ALP 活性(B)

Fig. 8 Cell proliferation (A) and ALP activity (B) of MC3T3E1 cells on the surface modified composites

(a) MPC; (b) MPC8; (c) MPC4; (d) MPCsb for different periods
表面改性复合材料表面在 SBF 中形成大量磷灰石, 这些磷灰石促进了成骨细胞分化 ${ }^{[17]}$ 。

\section{3 结论}

制备了 m-MCS/PK 复合材料, 采用砂纸打磨和 喷砂对其表面进行改性。表面改性不仅改变了复合 材料的表面形貌，提高了表面粗粘度和亲水性，而 且使其表面暴露出大量的 m-MCS 颗粒。喷砂处理, 在复合材料表面构建了多孔结构，其表面粗粘度和 亲水性明显大于砂纸打磨的复合材料表面，其表面 暴露出更多的 m-MCS 颗粒。表面改性复合材料在 SBF 中浸泡后, 其表面沉积出大量磷灰石, 表面改 性提高了复合材料的生物活性。细胞实验显示：表 面改性，特别是喷砂处理，促进了 MC3T3-E1 细胞 在复合材料表面粘附、增殖和分化。总之，表面改 性改变了复合材料表面的理化特征(如粗粘度、亲水 和多孔等)，因而提高了复合材料的生物学性能。

\section{参考文献:}

[1] OUYANG L, ZHAO Y, JIN G, et al. Influence of sulfur content on bone formation and antibacterial ability of sulfonated PEEK. Biomaterials, 2016, 83(4): 115-126.

[2] LU T, WEN J, QIAN S, et al. Enhanced osteointegration on tantalumimplanted polyetheretherketone surface with bone-like elastic modulus. Biomaterials, 2015, 51: 173-183.

[3] DAI C, GUO H, LU J, et al. Osteogenic evaluation of calcium/ magnesium-doped mesoporous silica scaffold with incorporation of rhBMP-2 by synchrotron radiation-based $\mu \mathrm{CT}$. Biomaterials, 2011, 32(33): 8506-8517.

[4] LIN K L, CHANG J, LU J X, et al. Preparation and properties of tricalcium phosphate/calcium silicate composite bioceramics. Journal of Inorganic Materials, 2006, 21(6): 1429-1434.

[5] XU A, LIU X, GAO X, et al. Enhancement of osteogenesis on micro/ nano-topographical carbon fiber-reinforced polyetheretherketonenanohydroxyapatite biocomposite. Materials Science \& Engineering $C$, 2015, 48: 592-598.

[6] XU S F, HU Y Y, LIN K L, et al. In vivo study of porous calcium silicate bioceramic in extra-osseous sites. Journal of Inorganic 
Materials, 2008, 23(3): 611-616.

[7] ZHU M, ZHANG J, ZHAO S, et al. Three-dimensional printing of cerium-incorporated mesoporous calcium-silicate scaffolds for bone repair. Journal of Materials Science, 2016, 51(2): 836-844.

[8] LI C, GAO L, CHEN F, et al. Fabrication of mesoporous calcium silicate/calcium phosphate cement scaffolds with high mechanical strength by freeform fabrication system with micro-droplet jetting. Journal of Materials Science, 2015, 50(22): 7182-7191.

[9] WU C, CHEN M, ZHENG T, et al. Effect of surface roughness on the initial response of MC3T3-E1 cells cultured on polished titanium alloy. Bio-Medical Materials and Engineering, 2015, 26: S155-S164.

[10] DENG Y, LIU X, XU A, et al. Effect of surface roughness on osteogenesis in vitro and osseointegration in vivo of carbon fiberreinforced polyetheretherketone-nanohydroxyapatite composite. International Journal of Nanomedicine, 2015, 10: 1425-1447.

[11] LAI Y H, KUO M C, HUANG J C, et al. On the PEEK composites reinforced by surface-modified nano-silica. Materials Science \& Engineering A, 2007, 458(1): 158-169.

[12] ZHU Y, ZHU M, HE X, et al. Substitutions of strontium in mesoporous calcium silicate and their physicochemical and biological properties. Acta Biomaterialia, 2013, 9(5): 6723-6731.

[13] HU G F, QUAN R F, CHEN Y M, et al. Fabrication, characterization, bioactivity, and biocompatibility of novel mesoporous calcium silicate/polyetheretherketone composites. RSC Advances, 2016, 6(62): 57131-57137.
[14] HOU J, ZHANG F, CHENG D, et al. Mineralization of a superficially porous microsphere scaffold via plasma modification. RSC Advances, 2017, 7(6): 3521-3527.

[15] MORAWSKA-CHOCHOL A, DOMALIK-PYZIK P, SZARANIEC B, et al. The effect of magnesium alloy wires and tricalcium phosphate particles on apatite mineralization on polylactide-based composites. Materials Letters, 2016, 180: 1-5.

[16] WANG Q X, WU Y Y, DONG X P, et al. Magnesium phosphate/ $\mathrm{PBS} /$ wheat protein biocomposite for bone repair. Journal of Inorganic Materials, 2015, 30(9): 957-962.

[17] DELIGIANNI D D, KATSALA N D, KOUTSOUKOS P G, et al. Effect of surface roughness of hydroxyapatite on human bone marrow cell adhesion, proliferation, differentiation and detachment strength. Biomaterials, 2000, 22(1): 87-96.

[18] TAKEDA I, SERIZAWA S, KANEKO A. Fabrication of microstructured scaffold using self-assembled particles and effects of surface geometries on cell adhesion. Mechanical Engineering Journal, 2016, 3(1): 1-8.

[19] DU H, WEI Z, WANG H, et al. Surface microstructure and cell compatibility of calcium silicate and calcium phosphate composite coatings on $\mathrm{Mg}-\mathrm{Zn}-\mathrm{Mn}-\mathrm{Ca}$ alloys for biomedical application. Colloids \& Surfaces B Biointerfaces, 2011, 83(1): 96-102.

[20] ITO T, SASAKI M, TAGUCHI T. Enhanced ALP activity of MG63 cells cultured on hydroxyapatite-poly (ethylene glycol) hydrogel composites prepared using EDTA-OH. Biomedical Materials, 2015, 10(1): 015025 . 